\title{
Perfiles sociodemográficos de la población ahorradora y acreditada en México, 2018'
}

\section{Sociodemographic profiles of the saving and accredited population in Mexico, 2018}

Daniela Sapien Vargas*1

Universidad Autónoma del Estado de México dansap0908@hotmail.com

Yuliana Gabriela Román Sánchez ${ }^{2}$

Universidad Autónoma del Estado de México ygromans@uaemex.mx

\section{Autor corresponsal.}

1 Universidad Autónoma del Estado de México, Facultad de Economía, C.P. 50100, Av. Paseo Tollocan 1541, Cerro de Coatepec, Toluca de Lerdo, MÉXICO.

2 Universidad Autónoma del Estado de México Centro de Investigación y Estudios Avanzados de la Población, C.P. 50100 Av. Paseo Tollocan 1541, Cerro de Coatepec, Toluca de Lerdo, MÉXICO.

\section{Resumen}

Propósito: El estudio busca definir perfiles sociodemográficos que identifican a las personas con ahorro formal e informal y aquellas que aplican para un crédito en México.

Diseño/metodología: Se trata de un estudio de carácter exploratorio, de corte transversal y con datos secundarios provenientes de la Encuesta Nacional de Inclusión Financiera (ENIF, 2018). Se estimó un análisis de correspondencias múltiples.

Resultados: La evidencia muestra que en el perfil sociodemográfico de la población con un ahorro o crédito formal sobresalen los hombres con educación superior, estabilidad laboral, ingresos altos y que tienen derecho a atención médica en instituciones como el IMSS e ISSSTE. Mientras que la población con ahorro o crédito informal está formada por adultos unidos y con poca estabilidad en un empleo y bajos ingresos.

Implicaciones: En los perfiles sociodemográficos de las personas con ahorro/ crédito formal e informal, las características sociales y laborales tienen mayor importancia que los aspectos individuales.

Limitaciones: Las características sociodemográficas consideradas son limitadas y captan solo un momento en el tiempo.

Originalidad/valor: La investigación aporta evidencias sobre las características sociodemográficas de la población con ahorro/crédito formal e informal, lo cual contribuye al conocimiento sobre la trayectoria del ciclo de vida y las decisiones tomadas del comportamiento planeado en México.
INFORMACIÓN ARTÍCULO

Recibido: 24 de Marzo 2021 Aceptado: 22 de Junio 2021

Palabras Claves:

Ahorros

Créditos

Educación financiera

Finanzas

México

1. El presente artículo representa un producto de la tesis "Análisis sociodemográfico de la cultura del ahorro y solicitudes crediticias en México, 2018" para la obtención del título de licenciado en Actuaria. Facultad de Economía de la Universidad Autónoma del Estado de México 


\begin{abstract}
Purpose: The study seeks to define sociodemographic profiles that identify people with formal and informal savings and those who apply for credit in Mexico.

Design/methodology: This is an exploratory study, cross-sectional and with secondary data from "Encuesta Nacional de Inclusión Financiera" (ENIF, 2018), a multiple correspondence analysis was estimated.

Results: The evidence shows that in the sociodemographic profile of the population with a savings or formal credit, men with higher education, with job stability, high income and who are entitled to receive medical care in institutions such as IMSS and ISSSTE stand out. While the population with informal savings or credit is made up by adults with little job stability and low income.

Implications: The social and labor characteristics of people with formal and informal savings/credit are of greater importance than individual aspects. Limitations: The sociodemographic characteristics considered are limited and captured only a moment in time.

Originality/value: The research provides evidence on the sociodemographic characteristics of the population with formal and informal savings/credit, thus, contributing to enlighten the life-cycle trajectory and the decisions made for planned behavior in Mexico.
\end{abstract}

\section{INTRODUCCIÓN}

La cultura del ahorro, la educación financiera y los comportamientos crediticios de una población son asuntos de gran interés y constante preocupación por parte de las autoridades de un país, pues en gran medida estas cuestiones forman parte de los pilares de una economía y permiten garantizar el bienestar de la población.

Para el caso particular de México, estos tópicos han sido tratados en varias ocasiones por parte de diferentes mandatarios o asociaciones, y se han intentado poner en marcha acciones que aporten una mejora en su funcionamiento, así como en la implementación de una cultura y educación financiera de la población; sin embargo, aún faltan acciones que garanticen que México está en un nivel óptimo de ahorro, inversión y crédito, que permita tener la tranquilidad y posible estabilidad económica de la población ante cualquier circunstancia.

El objetivo del presente estudio consiste en definir perfiles sociodemográficos que identifican a la población según su cultura de ahorro, y aqueIla que aplica para un crédito formal o informal, a partir del método de Análisis de Correspondencias Múltiples con datos de la Encuesta Nacional de Inclusión Financiera (ENIF, 2018) en México. Se plantea la pregunta de investigación ¿Qué carac206 terísticas sociodemográficas como la edad, sexo, nivel de escolaridad, estado conyugal y situación laboral, entre otras, presenta la población ante el ahorro y el crédito? En este sentido, se considera como hipótesis inicial que la población que ahorra es aquella con niveles de estudio más altos, conocimientos básicos de educación financiera, y en su mayoría hombres. Mientras que las personas que aplican solicitudes crediticias son jóvenes con un ingreso bajo, estudios básicos y de sexo femenino.

Los datos utilizados para esta investigación provienen de la ENIF (2018) que realizó el Instituto Nacional de Estadística y Geografía (INEGI). Por su parte, el Análisis de Correspondencias Múltiples es un método utilizado en la búsqueda de las características sociodemográficas de la población objetivo, para definir los perfiles que muestra la asociación estadística de variables.

El artículo se encuentra estructurado en cuatro secciones, además de esta introducción. La primera sección muestra el enfoque teórico que se tomó como referencia para este estudio, la segunda expone los antecedentes del tema, la tercera detalla la metodología utilizada, y la cuarta presenta y describe los resultados obtenidos. De esta forma, fue posible encontrar algunas conclusiones e incluso propuestas de solución. 


\section{ENFOQUE TEÓRICO}

En la teoría económica el ahorro es percibido como la oferta del capital, el cual es determinante en la productividad del trabajo y su evolución. Además, es considerado como un acto social de gran utilidad que ayuda a generar inversión y crecimiento. Es por ello, que, el ahorro es un tema con gran importancia y susceptible a investigaciones. Asimismo, existen teorías económicas que intentan explicar la posible relación que hay entre el ingreso, el consumo y el ahorro, y son varios los autores que a lo largo del tiempo han buscado y planteado proposiciones sobre el tema, como Keynes, Fisher, Modigliani, entre otros (Martínez, 2014).

Para el caso de Fisher, con la teoría microeconómica de la elección intertemporal, este plantea que un individuo recibirá una utilidad de aquellos activos acumulados al inicio del año, además del consumo presente y futuro (Martínez, 2014). Por otro lado, Keynes supone una relación entre el consumo y el ahorro de un individuo, con una renta disponible y un consumo constante o decreciente, esto dependiendo de la renta; sin embargo, los agentes no tienen una visión futura, pues las decisiones son tomadas solo considerando el presente (Lera, 1996).

Como base la teoría Keynesiana, y en un intento de ampliarla, surge la hipótesis de la renta permanente y la hipótesis del ciclo vital, ambas sobre una misma base teórica: un agente racional optimizador, un concepto de beneficio que será durable a lo largo de la vida de la persona y el consumo como una función igual a lo largo del tiempo, a la vez que se define de un mejor modo al incluir los servicios de los bienes duraderos, aunque no la adquisición de estos (Lera, 1996).

De esta forma, con algunas teorías e hipótesis planteadas de manera general, se hace especial énfasis en la hipótesis del ciclo vital, misma que es planteada por Modigliani y Brumberg (Ruiz, 2003):

La teoría del ciclo vital hace especial énfasis en el dinero ahorrado y trata de entender el proceder de los ahorros de cada individuo, partiendo de que el propósito de ahorrar a lo largo del ciclo de vida de una persona es mantener un estándar de vida razonablemente fijo. Como los ingresos personales típicamente suben en los años de juventud, alcanza su pico en la madurez y comienza a declinar, hasta llegar a ser muy bajos en el retiro, el deseo de mantener un consumo relativamente fijo conduce, según Modigliani, a que la gente joven ahorre y los ancianos desahorren (p.26).

Con lo anterior, es posible interpretar que la teoría o hipótesis del ciclo vital está basada en un modelo de optimización intertemporal que ayuda a predecir el comportamiento que tiene un individuo en el consumo a lo largo de su vida, es decir, la persona deberá planificar el consumo y el ahorro que sea necesario para poder lograr una estabilidad durante aquellos años en los que ya no tendrá ningún ingreso laboral. La principal restricción del ciclo de vida será, entonces, que el consumo actual que tenga el individuo no podrá ser superior al valor presente de sus ingresos, logrando así que el ahorro sea un medio para transferir los recursos a lo largo de diferentes etapas de su vida y los niveles de consumo actuales serán dependientes de los ingresos generados a lo largo de la vida (Moscoso, 2008).

En efecto, el individuo buscará maximizar la utilidad derivada de su consumo y, además, consciente de que cuando su vida activa termine, sus entradas de dinero se verán reducidas en gran medida, realizará el ahorro correspondiente para mantener el consumo en el momento de su jubilación. Pero aún existe una cuestión que queda inconclusa en la teoría, siendo esto el hecho de ¿cómo la persona decidirá distribuir su consumo a lo largo de su vida?, lo cual es respondido con el modelo que realiza un supuesto donde el individuo mantendrá un estilo de vida estable, logrando así cambios en su vida donde presente extremos de riqueza, es decir, que no pase de extrema escasez a extrema abundancia en el consumo. Para Moscoso (2008), La teoría del Ciclo de Vida plantea que,

Cuando la persona es joven, sus ingresos son menores al nivel de consumo deseado (o el nivel obtenido en la maximización), por lo que generalmente se endeudan, pues saben que su nivel de ingreso aumentará junto con su edad. Luego al llegar a adultos sus ingresos son mayores y les son suficientes para su consumo, pagar sus deudas de periodos anteriores y ahorran para cuando si vida laboral termine. Al jubilarse, su ingreso laboral cae a cero y deben consumir de sus ahorros anteriores (p.7).

Con lo anterior, es posible señalar que, cuando la renta del individuo es constante, su tasa de ahorro será constante y positiva a lo largo de su vida, excepto en el momento de la jubilación, momen- 
to en el cual será presentando un desahorro. El máximo nivel de ahorro es presentado en aquellas edades previas al retiro laboral, que a su vez coincide con el suceso de que los hijos parten de casa a realizar su vida y familia propia, y las rentas caen notablemente debido a que las pensiones son más bajas que los salarios (Lera, 1996).

No obstante, parte de las críticas realizadas a dicha teoría destaca que el agente económico no solo mira para sí, sino que este también ve por el bien se sus seres queridos, su familia y hogar, lo cual lleva a un nuevo análisis a la teoría que es denominada Modelo Intergeneracional, en el que las relaciones y transferencias entre generaciones tienen un gran peso de análisis e importancia (Lera, 1996).

Asimismo, otra crítica relevante se presenta desde la perspectiva de género, la cual representa una variable importante en un análisis sociodemográfico de la economía de las personas. Estudios destacan que a lo largo del ciclo de vida existe una diferencia en la trayectoria laboral entre hombres y mujeres. Al respecto la teoría propuesta por Modigliani muestra que la curva de ingreso tiene forma de una $U$ invertida; sin embargo, para el caso de las mujeres podría ser distinto, y "una posible fuente de diferencias de género en el bienestar económico es la diferencia en la capacidad de combinar con éxito el trabajo y las responsabilidades familiares para alcanzar los objetivos deseados en ambas áreas" (Villeda y Nava, 2019, p.6).

Entonces, a diferencia de la teoría del ciclo vital, para el caso de las mujeres la curva del ingreso podría ser distinta, pues en este caso en particular la curva no sería de U invertida, sino que sería en forma de una $M$, que representa su entrada a la vida laboral y posteriormente su retiro representado con el nacimiento de un hijo y finalmente su reincorporación cuando el hijo menor ya no es por completo dependiente. Pero esto podría tener otra variante que será que la participación femenina puede tener un perfil de un solo pico, presentado en el caso de que las mujeres no vuelvan a integrarse al mercado laboral (Villeda y Nava, 2019).

Para complementar y entender este tema, surgió la perspectiva teórica de la conducta planificada propuesta por Ajzen (1985), como evolución de la teoría de la acción razonada (TRA). Esta teoría tiene como hipótesis inicial el acto racional basado en el supuesto de que el ser humano actúa de manera lógica, considera toda la información disponible, calcula directa e indirectamente el impacto de las acciones que realiza. Las bases de esta teoría fueron los referentes para explicar las finanzas conductuales (Zaiunul, 2017).

La teórica de la conducta planificada se trata de un marco teórico que tiene como premisa explicar que la toma de decisiones individuales depende de tres variables, a saber: 1 ) actitud hacia el comportamiento, 2) normas subjetivas y 3 ) control de la conducta percibida. La primera se refiere al grado (positivo o negativo) del desempeño del comportamiento, mientras que la segunda hace referencia a la presión social percibida para participar o no en un comportamiento, en tanto la tercera considera las creencias sobre la presencia de factores de control que pueden facilitar u obstaculizar el comportamiento (Cucinelli, Gandolfi y Caia Soana, 2016).

En palabras de Schiffman y Kanuk (2004), Io anterior se refiere a explicar cómo los individuos toman decisiones para gastar sus recursos disponibles (tiempo, dinero, esfuerzo). Lo que incluye considerar las preguntas ¿qué compran? ¿por qué? ¿cuándo? ¿dónde? ¿cómo? ¿con qué frecuencia? ¿cómo lo evalúan? ¿el impacto sobre compras futuras? entre otras (Kowel, 2015). De lo anterior, Kowel (2015), concluye que los factores que influyen en el comportamiento son: la personalidad (que abarca características físicas y mentales), el estilo de vida (considera la forma en que las personas piensan, viven y se comportan) y la actitud monetaria (actitud del consumidor).

En este sentido, la teoría de la conducta planificada constituye un marco de referencia para comprender y predecir el comportamiento financiero de los individuos (Lajuni, Bujang y Lily, 2019), y da cabida al concepto de control percibido sobre las oportunidades, los recursos y las habilidades necesarias para realizar un comportamiento. Bajo la teoría de la conducta planificada, las actitudes se consideran un determinante en la intención del individuo de cambiar el comportamiento financiero, porque estas manifiestan el comportamiento específico del individuo, de gusto o disgusto; así como de una reacción positiva o negativa ante un evento (Lajuni y cols., 2019).

En este marco teórico, las creencias normativas y de comportamiento son las encargadas de transmitir mensajes financieros para alentar o desalentar a las personas a tomar decisiones financieras correctas o incorrectas, mientras que la norma social es la presión que influye en la intención de cambiar el comportamiento financiero del 
individuo (Lajuni y cols., 2019). En lo que respecta a las actitudes, Roberts y Jones (2001) destacan como hipótesis de Yamauchi y Templer's (1982), cuatro dimensiones relacionadas con la actitud hacia el dinero: 1) Poder-prestigio (las personas usan el dinero para impresionar o demostrar a otros un símbolo de éxito), 2) Retención-tiempo (dinero guardado en el presente para la vejez 0 simplemente realización de finanzas planificadas para el futuro), 3) Desconfianza (relacionada con la sensibilidad ante el cambio de los precios de buenos productos o servicios) y 4) Ansiedad (las personas ven el dinero como una fuente de ansiedad y de protección contra esta).

Desde una perspectiva más amplia, para Raiteri (2016), el comportamiento de los consumidores se puede dividir en factores: culturales, sociales, personales y psicológicos. Dentro de los culturales se encuentra la cultura, subcultura y clase social. En los sociales se ubican los grupos de referencia, familia, función y estatus. En los aspectos personales destaca la edad y etapa en el ciclo de vida, la ocupación y las circunstancias económicas, estilo de vida y personalidad. Respecto a los factores psicológicos sobresale la motivación, percepción, aprendizaje, actitudes y creencias. De esta forma, y con las diferentes teorías mostradas anteriormente, es posible entender que una decisión de consumo, gasto o ahorro no solo está determinada por factores exclusivamente económicos como hacen referencia las teorías del ciclo vital, sino que, además, esas decisiones comprenden factores psicológicos que son igualmente determinantes en dichas acciones.

\section{ANTECEDENTES}

Existen diversas investigaciones en el mundo sobre el tema analizado en este estudio. Lusardi y Mitchell (2011), realizaron un estudio sobre la educación financiera a nivel internacional, y dentro de sus hallazgos destaca que el analfabetismo financiero es generalizado, sin importar el desarrollo económico del país. Además, las personas que residen en países que han experimentado inflación, son quienes están más informados en dicho tema, y en todos los países la relación entre educación y educación financiera es fuertemente clara, a mayor educación mayor educación financiera. En lo que respecta a un análisis por razas, estos autores afirman que los blancos y los asiáticos son consistentemente más propensos a tener conocimientos financieros en comparación con los afroamericanos e hispanos.

Por su parte, Reddy, Bruhn y Tan (2013), realizaron un análisis sobre comportamientos, actitudes y conocimientos financieros, y mencionan que en comparación con encuestados de otros países como Armenia, Colombia, Líbano, Nigeria, Turquía y Uruguay, los mexicanos informaron tener un horizonte temporal relativamente más corto para la planificación financiera y no están acostumbrados a planear el modo en que gastarán su dinero. No obstante, en promedio, la población mexicana mostró puntajes más altos en la tendencia a ahorrar y a destinar fondos para gastos imprevistos de gran importancia (Reddy y cols., 2013).

Los resultados expuestos coinciden con otro estudio sobre educación financiera realizado por la Organización para la Cooperación y el Desarrollo Económico (OECD) en el año 2020. En el estudio participaron 26 países de tres continentes: Europa, Asia y América, este se realizó con la intención de conocer la opinión de los adultos sobre este tema. Dicho estudio consideró personas mayores de 18 años, entrevistando un total de 125787 adultos. Dentro de los resultados más relevantes se encontró que en general el nivel de educación financiera en los países resultó bajo. De manera particular destacó Hong Kong por ser el país con el nivel más alto de educación financiera, mientras que Italia resultó ser el más bajo (OECD, 2020).

En lo que respecta al uso de servicios financieros, el conocimiento acerca de ellos es elevado, pero el uso de estos es bajo, porque menos de la mitad de los encuestados compró un producto o servicio financiero. El 23\% de la muestra declaró recurrir a familiares o amigos para pedir prestado o ahorrar dinero (OECD, 2020). Solo una tercera parte tiene ahorrado lo equivalente a su ingreso de una semana, los jóvenes de entre 19 y 29 años corresponde a la población con menores conocimientos financieros y finalmente las personas que hacen uso de servicios financieros digitales tienen un mayor puntaje en conocimiento financiero (OECD, 2020).

Para el caso de México, estudios anteriores reveIan que la cultura del ahorro es escasa. Ejemplo de ello es que para el año 2012, 40\% de la población no tiene una cultura del ahorro y aunque pudiera parecer esperanzador que $60 \%$ de la población sí ahorra, la gran mayoría de esta lo hace de manera informal, es decir, a través de tandas o debajo del colchón, donde el gran problema radica en el riesgo 
que se tiene, la probabilidad de pérdida es mucha y la posibilidad de recuperarlo poca. Aunado a esta situación, la población mexicana tiende a ahorrar a pequeños plazos solo para la cobertura de emergencias o metas personales (Flores, 2013).

De acuerdo con la literatura revisada, se ha encontrado que algunas de las problemáticas causantes del bajo porcentaje de población que ahorra es la falta de educación financiera e información, la informalidad en el ahorro o control financiero, e incluso la parte de la población no bancarizada. Con relación a ello, la ENIF del año 2015, muestra que,

sólo el 36.6\% de la población lleva un registro de sus gastos (63.8\% mentalmente, disminuyendo su confiabilidad). También menciona que 33.6 millones de personas, 44\% tiene una cuenta de ahorro y $41.2 \%$ algún servicio de ahorro para su retiro. De las personas que no tienen productos de ahorro o crédito, 50\% manifiesta que sus ingresos son insuficientes y a $11 \%$ no le interesa adquirir ningún instrumento (INCyTU, 2018, p.4).

Respecto a los factores sociodemográficos de estudios realizados en México, la edad no es una variable determinante en la tenencia de tarjetas, mientras que el nivel de escolaridad y el de ingresos sí lo son, pues estas variables son más altas en aquellos hogares que cuentan con una tarjeta bancaria, así como también en estas viviendas existe un mayor nivel de integrantes contratados en el sector formal (Castellanos y Garrido, 2010).

La literatura muestra que en México existe una gran apertura a los créditos no solo bancarios sino también de consumo de almacenes comerciales. Por ejemplo, en el estudio de la Encuesta Nacional de Ingresos y Gastos de los Hogares (ENIGH) del año 2006, se encontró que las familias poseen uno o dos plásticos de alguna institución financiera y existe una penetración de las instituciones que varía según la economía de los hogares (Castellanos y Garrido, 2010).

En el estudio de Reddy y cols. (2013), sobre comportamientos, actitudes y conocimientos financieros en México, casi la mitad de los encuestados (49\%) no usa ningún servicio financiero actualmente (formal o informal) y existe un alto grado de utilización de fuentes de ahorro y crédito informal. Lo anterior se debe a que $91 \%$ de los adultos mexicanos es responsable de algún aspecto de la gestión de las finanzas del hogar, ya sea pagar cuentas, decidir cómo gastar el dinero o planificar el presupuesto. De los encuestados que toman decisiones financieras en el hogar,
$46 \%$ corresponde a hombres y $54 \%$ a mujeres, aunque para este último grupo su contribución al ingreso familiar sea menor que la de los hombres.

Sobre la planificación financiera en México, Reddy y cols. (2013), afirman que aproximadamente un $70 \%$ de los mexicanos planifica cómo gastar el ingreso, pero solo el $41 \%$ lo hace en forma habitual y apenas un tercio se ajusta al presupuesto. Los hogares con mayores ingresos y los residentes de zonas urbanas tienen mayores probabilidades de controlar sus gastos detalladamente y hacer planes más exactos sobre las finanzas familiares. Las personas de menores ingresos, los ancianos y los que están empleados en el sector informal o aquellos con educación primaria, son especialmente vulnerables a las dificultades económicas. La estrategia más común es el endeudamiento informal (Reddy y cols., 2013).

Estudios de INEGI en el año 2018, muestran que alrededor del 61\% de la población mexicana contaba con al menos una tarjeta de crédito de alguna tienda departamental, mientras que $34 \%$ de la misma tenía al menos una tarjeta de crédito bancaria (INEGI, 2018a). Castellanos y Garrido (2010), afirman que Banco Azteca es uno de los que tiene mayor penetración con las tarjetas de crédito en México en los primeros tres deciles, en los subsecuentes BBVA y Banamex tienen mayor participación de mercado. Coppel resultó ser la más común en todos los deciles, con excepción del décimo. Wal-Mart, Famsa, Suburbia y Elektra tienen participaciones significativas en los primeros deciles. Mientras que Liverpool, Sears, Fábricas de Francia y el Palacio de Hierro, son más comunes en deciles de ingreso alto.

A partir de los datos de la ENIF (2018), se infiere que en México hay un acelerado avance tecnológico de la banca, como es el servicio bancario por celular; sin embargo, solo un $22 \%$ de la población hace uso de ello, mientras que $43 \%$ de la población sigue haciendo uso de las sucursales bancarias en el año 2018 (INEGI, 2018a).

En lo que respecta a productos financieros, donde se consideran las cuentas con bancos o instituciones financieras, créditos formales o cuentas para el retiro, el $68 \%$ de los encuestados declara tener alguno de ellos, y de esta población la mayoría se encuentra concentrada en la parte noroeste del país, además en gran parte, es el hombre quien cuenta con alguno de estos productos (ENIF, 2018). Asimismo, datos de la ENIF (2018), permiten afirmar que disponer de una institución bancaria es el producto financiero más usado por 
parte de la población mexicana (dos de cada diez), pero esta posesión es generalmente por razones de pago de nómina o de pensiones, es decir, por la necesidad de recibir un pago en específico. En contraste a esto, el porcentaje de población que tiene una cuenta por razones de ahorro es notablemente más baja (13.5\%), pues esta cuenta es meramente por decisión de cada persona.

Respecto a créditos formales, los datos de la ENIF (2018) muestran que la región noroeste sigue siendo la región con mayor porcentaje de población que declara tener algún crédito formal (40\%) y que sigue siendo el sexo masculino el predominante en la tenencia de ellos (41\%). Dentro de la tenencia de tarjetas de crédito, son las provenientes de tiendas departamentales o autoservicios las que en su mayoría tiene la población (15.1\%) contra escasas tarjetas bancarias (8.3\%), por lo que sería relevante hacer un acercamiento a las normas de otorgamiento o posibles razones de la predominancia de las primeras.

\section{METODOLOCIA}

La presente investigación corresponde a un estudio trasversal con datos secundarios, llevado a cabo en cuatro fases. La primera consistió en obtener, revisar y limpiar los datos de la ENIF (2018). En la segunda se identificó la población objetivo. En la tercera se recodificaron y crearon las variables sociodemográficas. Y, en la cuarta fase se estimó el Análisis de Correspondencias Múltiples (AMC). A continuación, se describe cada fase.

La primera fase consta de 132 preguntas estructuradas en 13 secciones. La población objetivo de la encuesta son aquellas personas de entre 18 y 70 años, tomando una muestra de 14500 viviendas con una cobertura nacional y dividida en seis zonas geográficas para su estudio. Dicha selección de muestra se realizó con un diseño muestral probabilístico, estratificado y por conglomerados, donde la unidad de análisis es vivienda (INEGI, 2018b).

En la segunda fase se creó una nueva variable denominada población objetivo, la cual permitía identificar a las personas con un crédito formal o informal, y población que contaba con un ahorro formal o un ahorro informal. Para dicha clasificación se consideraron las respuestas a diferentes rubros, en los que, para el caso de créditos, considera los medios por los cuales se obtuvo dinero extra, y para el caso de ahorro el modo usado para la guarda de dinero (ver Figura 1).

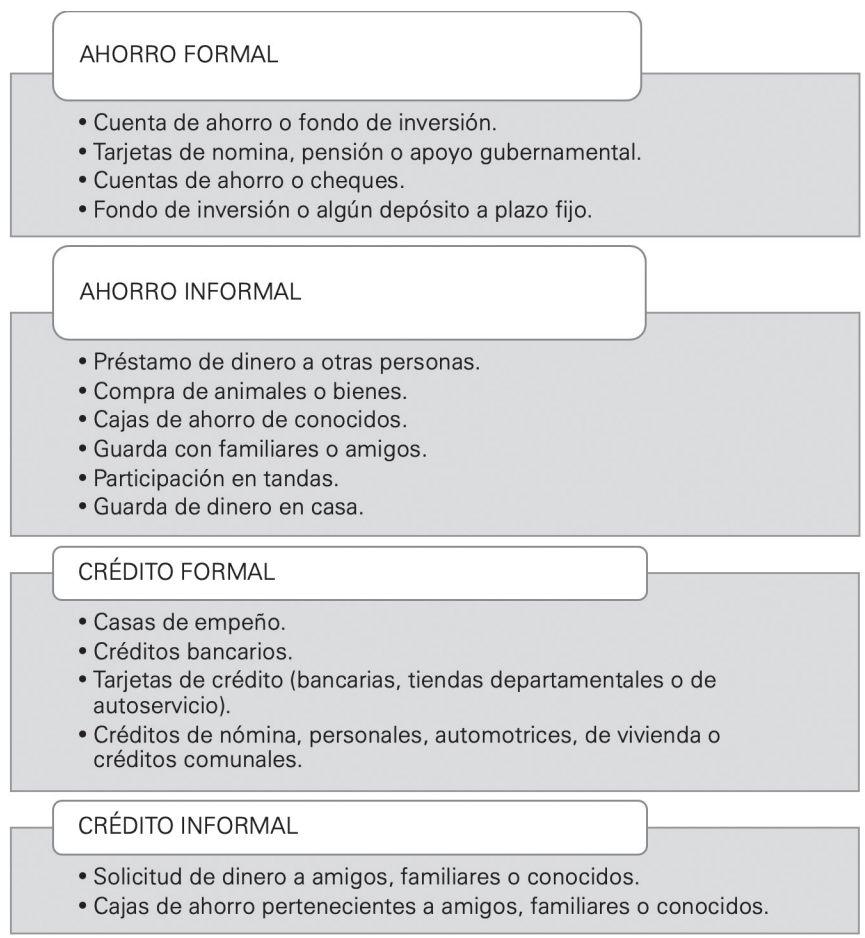

Figura 1. Rubro por grupos de población objetivo. Fuente: elaboración propia con información de la ENIF (2018). 
La población objetivo ascendió a 19593 casos, de nalmente $22.0 \%$ en crédito informal (ver Figura estos el $16.5 \%$ se ubicó en ahorro formal, $39.0 \% \quad 2$ 2). En él se puede observar el total de la muestra en ahorro informal, $22.5 \%$ en crédito formal, y fi- para hombres y mujeres.

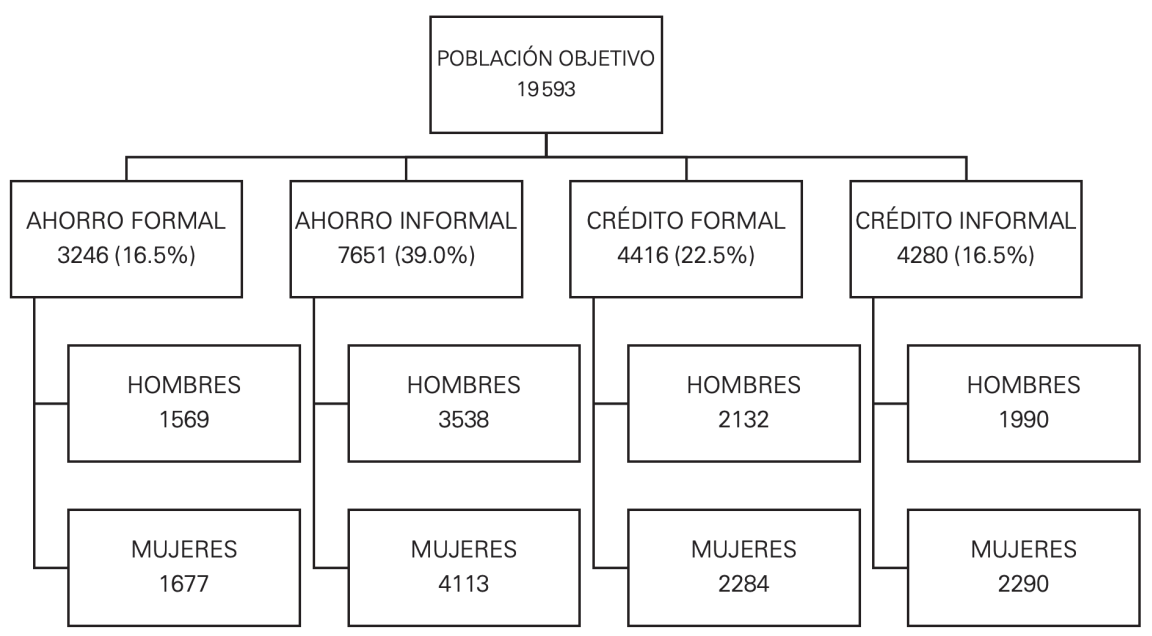

Figura 2. Clasificación grupal y cantidad de población objetivo. Fuente: elaboración propia con información de la ENIF (2018).

En la tercera fase, la manipulación, renombramiento, recodificación y agrupación de variables R Studio en su versión libre. Las variables se puefue realizada en el software de programación de den observar en la Tabla 1.

Tabla 1. Variables sociodemográficas para Análisis de Correspondencia Múltiple.

\begin{tabular}{|c|c|c|}
\hline Variable & Codificación & Escala \\
\hline Edad & $\begin{array}{l}\text { 0. Jóvenes } \\
\text { 1. Adulto } \\
\text { 2. Adulto mayor }\end{array}$ & Ordinal \\
\hline Sexo & $\begin{array}{l}\text { 0. Mujer } \\
\text { 1. Hombre }\end{array}$ & Nominal \\
\hline Nivel de escolaridad & $\begin{array}{l}\text { 0. Sin estudios } \\
\text { 1. Básica } \\
\text { 2. Media superior } \\
\text { 3. Superior y posgrado }\end{array}$ & Ordinal \\
\hline Situación conyugal & $\begin{array}{l}\text { 0. Unido } \\
\text { 1. Nunca unido } \\
\text { 2. Alguna vez unido }\end{array}$ & Nominal \\
\hline Servicios médicos & $\begin{array}{l}\text { 0. IMSS } \\
\text { 1. ISSSTE } \\
\text { 2. PEMEX / SEDENA } \\
\text { 3. Privado } \\
\text { 4. Otro } \\
\text { 5. No tiene }\end{array}$ & Nominal \\
\hline Ingreso laboral & $\begin{array}{l}\text { 0. No recibe } \\
\text { 1. Hasta } 100.67 \text { USD } \\
\text { 2. De } 100.72 \text { USD a } 503.36 \text { USD } \\
\text { 3. De } 503.41 \text { USD a } 1258.41 \text { USD } \\
\text { 4. Más de } 1258.46 \text { USD }\end{array}$ & Ordinal \\
\hline Urgencia económica & $\begin{array}{l}\text { 0. Sí } \\
\text { 1. No }\end{array}$ & Nominal \\
\hline Registro de gastos & $\begin{array}{l}\text { 0. Registro } \\
\text { 1. No registro }\end{array}$ & Nominal \\
\hline
\end{tabular}

Fuente: elaboración propia. 
La cuarta fase consistió en estimar un AMC con la intención de identificar los perfiles sociodemográficos. Este método consiste en la descripción e interpretación de datos por medio de un gráfico geométrico (Fernández, 2002). El AMC tiene como objetivo encontrar similitudes o semejanzas entre los individuos desde el análisis de un conjunto de variables, busca comparar a los individuos por la presencia o ausencia de elecciones de cada uno
(Husson, Lê y Pagès, 2013).

De manera general, el procedimiento consiste en formar una tabla disyuntiva (ver Figura 3) en la que se plasmen tantas columnas como categorías se tengan, que en caso de existir una variable continua esta deberá transformarse en nominal, ordenándose en intervalos con un rango de valores (De la Fuente, 2011).

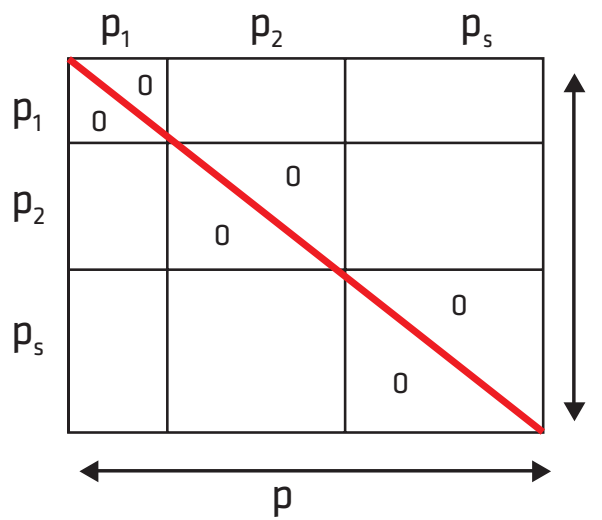

Figura 3. Tabla de Burt. Fuente: De la Fuente (2011).

De lo anterior, una variable representa ítems o individuos y el resto son variables cualitativas u ordinales que representan cualidades (De la Fuente, 2011). Para el caso del ACM, su número máximo de dimensiones estaría dado por:

$$
\operatorname{Min}\{\mathrm{m}, \mathrm{N}-1\}
$$

En la que $m$ representa el número de categorías de las variables y $\mathrm{N}$ es el tamaño de la muestra estudiada (López, 2006).

El $A M C$ fue realizado en el paquete estadístico Stata 64 versión 14. A continuación, se muestran los resultados obtenidos para el primer grupo de análisis y se encuentra que las variables con mayor porcentaje de inercia es la variable de jóvenes en los grupos de edades y la variable de nivel educativo en la clasificación superior y posgrado, en las cuales al tener el mayor porcentaje de inercia se sabe que son estas las variables con mayor dependencia y, en consecuencia, con mayor dispersión (ver Tabla 2).

Para el caso del segundo grupo, las variables con mayor inercia se encuentran en los grupos de acceso a servicios médicos y el nivel de ingreso laboral, por lo que se puede decir que son estas variables las que tienen mayor dependencia (ver Tabla 3).

\section{RESULTADOS}

En una primera etapa se estimó el AMC, y en esta se incluyeron solo variables sociodemográficas que pueden permitir una mejor caracterización de la población, como es la edad, el sexo, el nivel educativo y la situación conyugal. En segundo lugar, se incluyeron variables socioeconómicas y laborales como la situación laboral, los servicios de salud, el control de registros, el nivel de ingresos y la posibilidad de pago ante urgencias económicas.

De manera general, fue posible detectar variables influyentes y caracterizar cada uno de los grupos de la población objetivo, como entender parte de las decisiones tomadas por la población. A continuación, se describen los perfiles sociodemográficos de la población objetivo (ver Figura 4).

Población con ahorro formal (cuadrante I). Se puede observar que en su mayoría corresponde a hombres con educación superior o posgrado. En este sentido, se puede describir como un grupo privilegiado que además de sus gastos tiene la posibilidad de ahorrar de manera formal, a través de cuentas de ahorro, fondos de inversión, guardando dinero en tarjetas de nómina, cuentas de cheques o de algún depósito a plazo fijo. 
Tabla 2. Resultados estadísticos primer grupo.

\begin{tabular}{|c|c|c|c|c|c|c|c|c|c|}
\hline \multirow{2}{*}{ Categorías } & \multicolumn{3}{|c|}{ General } & \multicolumn{3}{|c|}{ Dimensión 1} & \multicolumn{3}{|c|}{ Dimensión 2} \\
\hline & Masa & Cal. & Iner. (\%) & Coord. & Corr. & Cont. & Coord. & Corr. & Cont. \\
\hline \multicolumn{10}{|l|}{ Población objetivo } \\
\hline A. Formal & 0.033 & 0.982 & 0.008 & 0.084 & 0.365 & 0.004 & 0.110 & 0.617 & 0.018 \\
\hline A. Informal & 0.078 & 0.924 & 0.003 & -0.002 & 0.001 & 0.000 & -0.055 & 0.923 & 0.011 \\
\hline C. Formal & 0.045 & 0.906 & 0.004 & -0.020 & 0.052 & 0.000 & 0.079 & 0.854 & 0.013 \\
\hline C. Informal & 0.044 & 0.912 & 0.004 & -0.041 & 0.253 & 0.001 & -0.067 & 0.660 & 0.009 \\
\hline \multicolumn{10}{|l|}{ Edad } \\
\hline Jóvenes & 0.056 & 0.998 & 0.239 & 0.464 & 0.651 & 0.226 & -0.339 & 0.347 & 0.294 \\
\hline Adultos & 0.128 & 0.979 & 0.064 & -0.152 & 0.594 & 0.055 & 0.122 & 0.385 & 0.087 \\
\hline A. Mayores & 0.016 & 0.886 & 0.053 & -0.429 & 0.699 & 0.054 & 0.222 & 0.187 & 0.035 \\
\hline \multicolumn{10}{|l|}{ Sexo } \\
\hline Hombres & 0.094 & 0.270 & 0.004 & 0.026 & 0.227 & 0.001 & 0.012 & 0.043 & 0.001 \\
\hline Mujeres & 0.106 & 0.270 & 0.003 & -0.024 & 0.227 & 0.001 & -0.010 & 0.043 & 0.001 \\
\hline \multicolumn{10}{|l|}{ Nivel educativo } \\
\hline Básica & 0.099 & 0.999 & 0.183 & -0.343 & 0.814 & 0.216 & -0.264 & 0.185 & 0.120 \\
\hline Media Sup. & 0.047 & 0.982 & 0.018 & 0.141 & 0.684 & 0.017 & -0.093 & 0.298 & 0.018 \\
\hline $\begin{array}{r}\text { Superior y pos- } \\
\text { grado }\end{array}$ & 0.055 & 1.000 & 0.274 & 0.499 & 0.638 & 0.253 & 0.376 & 0.362 & 0.350 \\
\hline \multicolumn{10}{|l|}{ Situación conyugal } \\
\hline Unido & 0.126 & 0.793 & 0.014 & -0.082 & 0.778 & 0.016 & 0.011 & 0.015 & 0.001 \\
\hline Nunca Unido & 0.044 & 0.987 & 0.095 & 0.392 & 0.913 & 0.126 & -0.111 & 0.074 & 0.025 \\
\hline Alguna vez unido & 0.030 & 0.766 & 0.033 & -0.232 & 0.610 & 0.030 & 0.0117 & 0.155 & 0.018 \\
\hline
\end{tabular}

Cal.: “Calidad”, Iner.: “Inercia”, Coord.: “Coordenadas”, Corr.: “Correlación”, Cont.: “Contribución”. Fuente: elaboración propia con información de la ENIF (2018).

Fuente: elaboración propia con información de la ENIF (2018)

Estos resultados encontrados coinciden con los hallados por Reddy y cols. (2013), así como aqueIlos por la Comisión Nacional Bancaria y de Valores (CNBV) para el año 2018. Ambos estudios afirman que el uso de productos financieros formales es más común en el caso de los hombres, sobre todo aquellos que residen en zonas urbanas, con altos ingresos y son empleados del sector formal. (Reddy y cols., 2013; CNBV, 2021a).

Población con ahorro informal (cuadrante II). Este grupo está formado por adultos unidos (casados o en unión libre) o alguna vez unidos (separados, divorciados o viudos). En este sentido, se puede apreciar que, al estar unidos seguramente con hijos, los gastos se incrementan y queda la posibilidad de un ahorro informal para completar los gastos a corto tiempo, lo anterior se lleva a cabo a través de tandas, ahorro abajo del colchón, cajas de ahorro, entre otros.
El nivel educativo es uno de los aspectos más analizados en las diversas investigaciones sobre el ahorro y crédito. Al respecto, se ha encontrado evidencia que un nivel educativo bajo junto a un ingreso bajo, se asocia con una mayor probabilidad de tener que pedir prestado de manera informal. Respecto al nivel de endeudamiento, Reddy y cols. (2013) destacan que el $29 \%$ de los encuestados mexicanos informó que tenía una deuda con un amigo o un miembro de la familia. El 30\% de los encuestados dijo que habitual u ocasionalmente pedía prestado para comprar alimentos u otros artículos de primera necesidad, y $43 \%$ informó que pedía dinero para saldar deudas (Reddy y cols., 2013).

Por su parte, la CNBV (2021b) agrega que los instrumentos más utilizados en el financiamiento informal fueron los familiares (24\%), amigos 0 conocidos (15\%), caja de ahorro del trabajo o de 
Tabla 3. Resultados estadísticos segundo grupo.

\begin{tabular}{|c|c|c|c|c|c|c|c|c|c|}
\hline \multirow{2}{*}{ Categorías } & \multicolumn{3}{|c|}{ General } & \multicolumn{3}{|c|}{ Dimensión 1} & \multicolumn{3}{|c|}{ Dimensión 2} \\
\hline & Masa & Cal. & Iner. (\%) & Coord. & Corr. & Cont. & Coord. & Corr. & Cont. \\
\hline \multicolumn{10}{|l|}{ Población objetivo } \\
\hline A. Formal & 0.034 & 0.988 & 0.031 & 0.188 & 0.822 & 0.028 & 0.085 & 0.166 & 0.082 \\
\hline A. Informal & 0.076 & 0.798 & 0.006 & -0.048 & 0.610 & 0.004 & 0.027 & 0.188 & 0.018 \\
\hline C. Formal & 0.046 & 0.791 & 0.009 & 0.082 & 0.693 & 0.007 & -0.031 & 0.098 & 0.015 \\
\hline C. Informal & 0.044 & 0.964 & 0.027 & -0.149 & 0.748 & 0.022 & -0.080 & 0.217 & 0.095 \\
\hline \multicolumn{10}{|l|}{ Servicio médico } \\
\hline IMSS & 0.081 & 0.975 & 0.058 & 0.181 & 0.965 & 0.062 & -0.018 & 0.009 & 0.008 \\
\hline ISSSTE & 0.015 & 0.994 & 0.128 & 0.618 & 0.932 & 0.132 & -0.159 & 0.061 & 0.126 \\
\hline Pemex Sedena & 0.001 & 0.488 & 0.016 & 0.593 & 0.472 & 0.008 & 0.110 & 0.016 & 0.004 \\
\hline Privado & 0.002 & 0.863 & 0.012 & 0.502 & 0.850 & 0.011 & 0.060 & 0.012 & 0.002 \\
\hline No tiene & 0.101 & 0.997 & 0.138 & -0.252 & 0.977 & 0.149 & 0.035 & 0.019 & 0.042 \\
\hline \multicolumn{10}{|l|}{ Ingreso laboral } \\
\hline No recibe & 0.001 & 0.300 & 0.009 & -0.246 & 0.143 & 0.001 & 0.257 & 0.156 & 0.023 \\
\hline $2 \mathrm{~m}$ & 0.107 & 0.998 & 0.168 & -0.272 & 0.992 & 0.184 & 0.021 & 0.006 & 0.016 \\
\hline $10 \mathrm{~m}$ & 0.071 & 0.993 & 0.098 & 0.249 & 0.936 & 0.102 & -0.061 & 0.056 & 0.089 \\
\hline $25 \mathrm{~m}$ & 0.018 & 0.983 & 0.115 & 0.543 & 0.970 & 10.123 & 0.062 & 0.013 & 0.023 \\
\hline$+25 \mathrm{~m}$ & 0.003 & 0.945 & 0.029 & 0.589 & 0.848 & 0.027 & 0.199 & 0.097 & 0.045 \\
\hline \multicolumn{10}{|l|}{ Urgencia económica } \\
\hline Sí & 0.102 & 0.975 & 0.038 & 0.110 & 0.069 & 0.029 & 0.071 & 0.288 & 0.174 \\
\hline No & 0.098 & 0.975 & 0.039 & -0.114 & 0.687 & 0.029 & -0.074 & 0.288 & 0.179 \\
\hline \multicolumn{10}{|l|}{ Registro de gastos } \\
\hline Registro & 0.086 & 0.986 & 0.045 & 0.153 & 0.940 & 0.047 & 0.034 & 0.045 & 0.033 \\
\hline No registro & 0.114 & 0.986 & 0.034 & -0.115 & 0.940 & 0.035 & -0.025 & 0.045 & 0.025 \\
\hline
\end{tabular}

Cal.: “Calidad”, Iner.: "Inercia”, Coord.: “Coordenadas", Corr.: “Correlación”, Cont.: “Contribución”. Fuente: elaboración propia con información de la ENIF (2018).

Fuente: elaboración propia con información de la ENIF (2018)

conocidos (7\%) y una casa de empeño (5\%). Aproximadamente, 19.2 millones de personas adultas piden dinero prestado a sus familiares para imprevistos. Asimismo, al considerar la edad de los individuos, los hallazgos muestran que las personas adultas empleadas en el sector informal, con educación primaria, son especialmente vulnerables a las dificultades económicas (Reddy y cols., 2013). Situación que coincide con los resultados de la presente investigación.

Población con crédito formal (cuadrante III). Se observa que prevalecen las mujeres con educación básica. En ese grupo se pudo observar que son las mujeres con baja escolaridad las que acceden a créditos bancarios o de nómina, o a través de tarjetas pertenecientes a instituciones bancarias 0 incluso a tiendas departamentales, donde los intereses suelen ser altos, pues al no contar con un trabajo formal bien remunerado, las instituciones incrementan los costos para que las personas accedan al crédito. En lo que respecta a la solicitud de estas, el proceso es sencillo. En algunos casos no piden comprobantes ni cuestionan los ingresos, simplemente otorgan las tarjetas con algún comprobante domiciliario e identificación oficial. Situación que coincide con la investigación de Oliva y Oropeza (2012):

En México se ha dado mayor apertura a los créditos no solo en instituciones bancarias, sino también en almacenes comerciales que abastecen los requerimientos de la familia en alimentos, ropa, electrodomésticos, en- 
tre otros. Este tipo de ventas se anuncian de manera desmesurada en medios de comunicación en los que se da a entender el disfrute de muchas facilidades y pocos compromisos (p.57).

A partir de lo anterior, se puede asumir que la mujer puede prevalecer en la adquisición de créditos formales a través de las tarjetas de crédito de tiendas departamentales, pues son estas las que le ayudan a la adquisición de alimentos, ropa e insumos en general que son utilizados en casa.

Aunque en la presente investigación se encontró que las mujeres son quienes logran acceder a créditos formales, estos se refieren en mayor medida a los créditos de tiendas departamentales. Lo anterior hace contraste con la desventaja que las mujeres han evidenciado en diversas investigaciones, donde los resultados muestran que son aquellas que residen en zonas rurales y de grupos de bajos ingresos quienes utilizan más los préstamos de cajas de ahorro y fuentes informales de crédito y ahorro, como las tandas y los préstamos de familiares o amigos (Reddy y cols., 2013).

Población con crédito informal (cuadrante IV). Se ubica a la población nunca unida, es decir, los solteros con un nivel de escolaridad media superior y donde los jóvenes tienen mayor presencia. Este grupo está compuesto por la población más joven y sin compromisos, pues son solteros sin hijos y con escolaridad media. Ellos suelen pedir prestado a sus familiares, amigos o en cajas de ahorro.

Al respecto, la CNBV (2021b) encontró que el sobregasto o sobreendeudamiento es lo que influye para buscar créditos informales, en virtud de que aumenta $21 \%$ la probabilidad de tener financiamiento informal, así como percibir un ingreso mensual menor a 266.78 USD² mensuales $^{2}$ aumenta un $11 \%$ dicha probabilidad. En sentido contrario, los factores que disminuyen la probabilidad de tener financiamiento informal fueron tener empleo formal y residir en un área urbana.

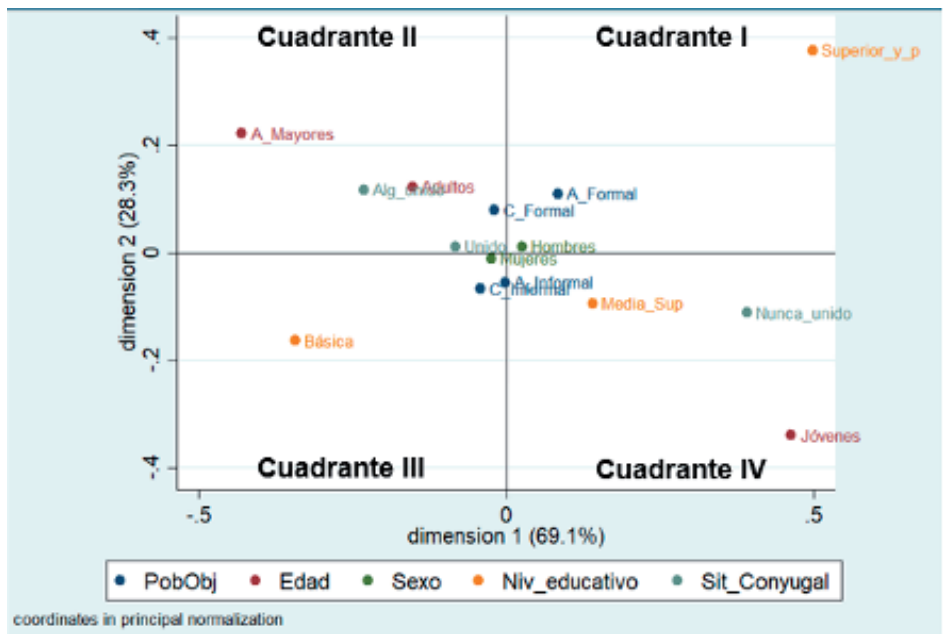

Figura 4. Población acreditada y ahorradora en México según características sociodemográficas, 2018. Fuente: elaboración propia con información de la ENIF (2018).

Al incluir variables socioeconómicas y laborales se obtuvieron los siguientes perfiles socioeconómicos:

Población con ahorro formal (cuadrante I). Se puede observar que este grupo de población lleva un registro de sus cuentas, tiene servicio de salud en instituciones privadas o instituciones pertenecien- tes a instituciones como Petróleos Mexicanos (PEMEX) o Secretaría de la Defensa Nacional (SEDENA), sus ingresos son de 1258.41 USD o más. Este grupo es privilegiado al ganar dicha cantidad y poder recibir atención médica en hospitales nivel 3 de PEMEX o SEDENA, donde se encuentran médicos especialistas.

2. Todas las cifras monetarias de dólares estadounidenses (USD) registradas en esta investigación son conversiones con tipo de cambio al 1 de junio del 2018 (BANXICO, 2021). 
El que dicha población tenga acceso a estos servicios de salud es sinónimo de personas que tienen un trabajo estable y de manera formal, un estilo de vida estable y de igual forma tienen la posibilidad de guardar dinero en instituciones bancarias. Este mismo perfil sociolaboral coincide con otros estudios, donde se destaca que los grupos de ingresos más altos usan más productos financieros formales que los otros grupos de ingresos, mientras que los grupos de menores ingresos están más habituados a pedir prestado a familiares 0 amigos (Reddy y cols., 2013).

Población con ahorro informal (cuadrante II). En este caso, la población no tiene derecho a recibir servicios de salud por parte de su trabajo, reciben ingresos bajos de alrededor de 100.67 USD o menos, e incluso se registran personas que no reciben. Representa un grupo en desventaja, pues al no acceder a la atención médica en instituciones públicas de población trabajadora, hacen que ellos desembolsen para recibirla y la situación se complica dado que sus ingresos son muy bajos. En este caso, son ellos quienes desembolsan cantidades de dinero para recibir un servicio médico, resguardando cantidades de dinero debajo del colchón, con amigos, en cajas de ahorro o incluso participando en tandas. De lo contrario, tendrían que endeudarse.

Estos resultados van de la mano con otros estudios. Por su parte, la CNBV (2021a) destaca que el ahorro informal y el ahorro formal no son mutuamente excluyentes y la probabilidad de posesión de una cuenta cuando se tiene un empleo formal aumenta en un $40 \%$ y del uso de tarjeta de débito en $20 \%$, además el crédito formal aumenta la probabilidad de ahorro formal. Asimismo, afirma que tener un crédito formal y llevar un presupuesto aumentan la probabilidad de tener ahorro informal ( $8 \%$ y $10 \%$, respectivamente) y formal (10\% y $7 \%$, correspondientemente). Por el contrario, el sobregasto actúa como una barrera para el ahorro formal e informal, porque disminuye su probabilidad en $9 \%$ y $10 \%$, respectivamente, así como para la tenencia de alguna cuenta en $3 \%$ y uso de tarjeta de débito en $4 \%$.

Población con crédito informal (cuadrante III). En este grupo se encuentran aquellos que no tienen registro de sus gastos, y que además si se les presentara alguna urgencia que implique una salida de dinero, no tendrían la capacidad de pago.

Parte de la problemática de dicha población es la falta de un control de sus ingresos y gastos, esto dada la falta del registro de estos, además, al no tener un control de los gastos los lleva a que ante alguna emergencia económica necesiten solicitar dinero por algún medio, y la manera más fácil de hacerlo es a través de un crédito informal con familiares, amigos o conocidos, quienes podrán otorgar un préstamo de manera sencilla y rápida.

Al respecto, la CNBV (2021b) confirma que el acceso al crédito informal está asociado de forma positiva con residir en alguna área rural y con ser hombre, por el contrario, el aumento en el nivel salarial y la edad se relacionan de forma negativa con este tipo de financiamiento. A su vez, las personas con educación media superior y con empleos informales registran una mayor tenencia de estos instrumentos, en comparación a sus contrapartes. La población reportó que el destino del crédito informal más frecuente son los gastos del hogar.

Población con crédito formal (cuadrante IV). Este conjunto de personas se caracteriza porque tiene derecho a recibir atención médica en el Instituto Mexicano del Seguro Social (IMSS), Instituto de Seguridad y Servicios Sociales de los Trabajadores del Estado (ISSSTE) u otro, y sus ingresos se encuentran alrededor de los 503.36 USD. Este grupo se considera de clase trabajadora que recibe sus prestaciones y puede acceder a créditos bancarios por su forma de comprobar ingresos.

Al ser personas con acceso a servicios médicos por parte del IMSS e ISSSTE, la mayoría tiene un trabajo estable en instituciones gubernamentales y privadas, y perciben un salario fijo y comprobable, es más fácil que accedan a un préstamo por medio de instituciones bancarias.

Similar perfil se encontró en el estudio de Reddy y cols. (2013), donde se afirma que un $81 \%$ de los afiliados al ISSSTE ahorra en bancos, mientras que solo $20 \%$ de quienes reciben beneficios sociales o de los afiliados al Seguro Popular tiene cuentas de ahorro en una institución financiera formal. De manera complementaria, agregan que los productos de ahorro informal como las tandas, son usadas en menor medida por los beneficiarios del IMSS y el ISSSTE.

Con los resultados presentados anteriormente, es posible encontrar similitud con la teoría del ciclo vital, en la cual queda evidenciado que la edad en la que se encuentran las personas es un factor importante en su economía y estabilidad, que a su vez tiene una repercusión en las instituciones, formalidad o informalidad que se tiene al mo- 


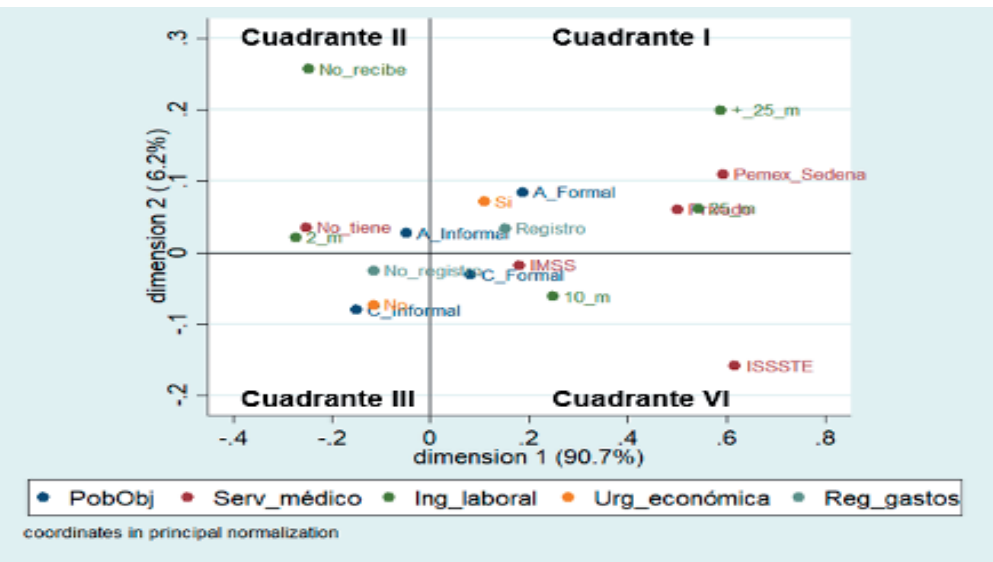

Figura 5. Población acreditada y ahorradora en México según aspectos socioeconómicos y laborales, 2018. Fuente: elaboración propia con información de la ENIF (2018).

mento de generar un ahorro o en la petición de un crédito monetario. En ese sentido, se pudo observar que los jóvenes tienen un perfil de desventaja frente a los adultos, que los coloca en situaciones de mayor vulnerabilidad económica al ser ellos quienes acceden en mayor proporción a créditos y ahorro informales.

Es fundamental recalcar que la edad juega un papel muy importante en la teoría del ciclo de vida y es una variable muy representativa, pues en la trayectoria de vida de cada individuo este tiene diferentes responsabilidades y actividades productivas que van de la mano con una variación de ingresos y egresos. En la niñez, por ejemplo, se tiene una economía totalmente dependiente de los padres, mientras que en la etapa adulta es el mismo individuo quien busca y trabaja por un ingreso propio, para, finalmente, en las edades avanzadas ya no laborar y depender totalmente de aquella riqueza creada con anterioridad.

No obstante, el manejo y adquisición correcto del ingreso no depende solo de la edad, por lo cual es importante que cada uno de los individuos sepa manejar correctamente su dinero, saber cuándo y cuánto es necesario gastar, así como mantener un estilo de vida de acuerdo con sus posibilidades; pero también mejorar las condiciones sociales, laborales y económicas para que se puedan cumplir los fundamentos de dicha teoría. Por lo anterior, no se puede afirmar que al incrementar la edad mejoran de facto las condiciones de ahorro y crédito de los individuos, porque además existen diferencias por sexo. Por consiguiente, esta investigación aporta elementos para sumar a la hipótesis que en la situación de ahorro y crédito influyen elementos individuales, pero también sociales y laborales en conjunto con la sociedad y la economía.

Por su parte, los fundamentos de la teoría de la conducta planificada permitieron entender que la decisión de ahorrar o endeudarse está relacionada con aspectos como el estilo de vida, el medio donde se desenvuelven las personas, el ambiente de trabajo, las relaciones individuales y sociales, los conocimientos sobre educación financiera, de llevar o no un registro de sus gastos. Todos aspectos que influyen en las decisiones de los individuos.

En definitiva, los resultados dejan de manifiesto que la población mexicana tiene una cultura del ahorro, pero que solo ahorra para compras específicas u objetivos a corto plazo. Además, que en su mayoría lo hace por medios informales como guardar el dinero debajo del colchón o a través de tandas y cajas de ahorro con amigos o conocidos, lo cual implica un alto riesgo de fraude y/o pérdida de su dinero.

Asimismo, los resultados arrojaron que cuando el hombre está casado, entonces es él quien solicita créditos en instituciones formales, mientras que, para los otros casos de situación conyugal, la mujer es quien predomina en dichas solicitudes. La tenencia de un trabajo en posiciones como patrón o empleado, van muy relacionadas con niveles educativos de nivel preparatorio o superior, que a su vez influye fuertemente en el nivel de ingreso alto y a las adquisiciones de créditos o cuentas de ahorro por medio de instituciones financieras. 
Es posible, entonces, mencionar que los mexicanos tienen una conducta planificada para ahorrar y acreditarse de acuerdo con su contexto social, económico y laboral.

\section{CONCLUSIONES}

A partir de los resultados encontrados en la presente investigación, es posible enunciar algunas conclusiones. La forma de controlar y administrar el dinero, la manera en cómo se utiliza en la vida diaria y la satisfacción de sus necesidades, son temas que forman parte y están presentes en las inquietudes de los investigadores para entender los comportamientos de las personas. Estas problemáticas han creado diferentes teorías que intentan explicar dichos comportamientos desde perspectivas económicas hasta psicológicas.

Después de haber realizado el AMC, se puede llegar a varias conclusiones: entre las más importantes es que las variables relacionadas con un ahorro formal y un crédito formal son muy similares, son personas quienes consideran tener un ingreso suficiente que les permite el pago de emergencias económicas, donde el ingreso percibido de manera quincenal o mensual con montos fijos mayores a 100.67 USD, el cual sea depositado vía nómina, tienen estudios arriba de nivel medio superior y ocupan puestos de empleado o patrón con acceso a servicios médicos privados o en instituciones públicas como IMSS, ISSSTE, PEMEX o SEDENA y, en general, no tienen dependientes económicos o muy pocos.

En el caso de las variables que representan a las personas con un crédito y ahorro informal, son personas que consideran no tener un ingreso suficiente y en consecuencia no se sienten capaces de tener capacidad financiera ante alguna emergencia económica, tienen un salario variable menor a 100.67 USD, el cual es dado de manera semanal y en efectivo. En esta perspectiva, se puede concluir que la población con crédito informal comparte similares características que aquella que ahorra de manera informal.

El análisis por sexo mostró que los hombres son quienes consideran ganar lo suficiente y tener una capacidad de pago ante alguna emergencia económica, ganan más de 100.67 USD de manera fija y con periodicidad quincenal o mensual, los cuales son entregados vía nómina, tienen puestos de empleado o patrón y un acceso a servicios

\section{REFERENCIAS}

Ajzen, I. (1985). From intentions to actions: A theory of planned behavior. In J. Kuhl \& J. Beckman (Eds.), Action-control: From cognition to behavior, (pp. 11-39). Heidelberg: Springer.

BANXICO (2021). Sistema de información económica. Recuperado de: https://www.banxico.org. $\mathrm{mx} /$ tipcamb/main.do?page=tip\&idioma=sp

Castellanos, S. G., y Garrido, D. (2010). Tenencia y uso de tarjetas de crédito en México. Un análisis de los datos de la Encuesta Nacional de Ingresos y Gastos de los Hogares 2006. El Trimestre Económico, 77 (305), 69-103.

CNBV. (2021a) El ahorro en México: productos, instrumentos y evolución. Estudios de inclusión financiera, 1-64.

CNBV. (2021b) El crédito en México: productos, instrumentos y evolución. Estudios de inclusión financiera, 1-58.

Cucinelli, D., Gandolfi, G., \& Gaia Soana, M. (2016). Customer and Advisor Financial Decisions: the Theory of Planned Behavior Perspective. International Journal of Business and Social Science, 7(12), 80-92.

De la Fuente, F. S. (2011). Análisis correspondencias simples y múltiples. Universidad Autónoma de Madrid, 58.

ENIF. (2018). Presentación de resultados. Encuesta Nacional de Inclusión Financiera, 1-23.

Fernández, O. F. (2002). El uso del Análisis de Correspondencia Simple (ACS) como ayuda en la interpretación del dato en arqueología. Un caso de estudio. Boletín Antropológico, 687-713.

Flores, M. (2013). ¿Cómo ahorramos los mexicanos? TU BOLSILLO, 36-38. Recuperado de: https://revista.condusef.gob.mx/PDF-s/2013/159/ mexicanos.pdf

Husson, F., Lê, S., y Pagès, J. (2013). Análisis de datos con R. Colombia: Escuela Colombiana de Ingeniería.

INCyTU. (2018). Educación financiera en México. NOTA-INCYTU; Información Científica y Tecnológica para el Congreso de la Unión, 18, 1-6. Recuperado de: https://www.foroconsultivo.org.mx/ INCyTU/documentos/Completa/INCYTU_18-018. pdf 
INEGI. (2018a). Encuesta nacional de inclusion financiera: Presentación de resultados. Recuperado de: https://www.inegi.org.mx/programas/ enif/2018/

INEGI. (2018b). Encuesta Nacional de Inclusión Financiera (ENIF) 2018: Diseño conceptual. Recuperado de: https://www.inegi.org.mx/programas/ enif/2018/

Kowel, C. A. (2015). The influence of personality, lifestyle, money attitude on customer purchase decision (Case study: Manado Grand Palace Convention Hall). Jurnal Berkala IImiah Efisiensi, 15(5), 417-425.

Lajuni, N., Bujang, I., \& Lily, J. (2019). Financial knowledge and modified theory of planned behaviour influence on financial behavioural intention: A multi-group analysis. The Business and Management Review, 10(3), 285-293.

Lera, L. F. (1996). Teorías macroeconómicas explicativas del ahorro de las economías domésticas: situación actual del debate. Cuadernos de Economía, 24, 91-117.

López, J. A. (2006). El análisis de correspondencias. Departamento Psicología Experimental, 28.

Lusardi, A., \& Mitchell, O. (2011). Financial literacy around the world: an overview. Journal of Pension Economics \& Finance, 10(4), 1-17.

Martínez, G. L. (2014). La hipótesis del ingreso permanente: un análisis para el caso de México. División de Ciencias Sociales y Humanidades.

Moscoso, C. A. (2008). Características del Ahorro en el Ciclo de Vida. Facultad de Economía y Negocios, Universidad de Chile, 1-23.

OECD (2020). OECD/INFE 2020 International Survey of Adult Financial Literacy, 1-78. Recuperado de: https://www.oecd.org/financial/education/ oecd-infe-2020-international-survey-of-adult-financial-literacy.pdf
Oliva, E., y Oropeza, K. (2012). Problemática de los intereses en créditos bancarios para la economía familiar. Inventio, la génesis de la cultura universitaria en Morelos, 57-60.

Raiteri, M. D. (2016). El comportamiento del consumidor actual. Universidad Nacional de Cuyo, FCE. Recuperado de: https://bdigital.uncu.edu. ar/objetos_digitales/8046/raiteri-melisa-daniela.pdf

Reddy, R., Bruhn, M., \& Tan, C. (2013). Financial capability in Mexico: results from a national survey on financial behaviors, attitudes and knowledge. Banco Mundial, Washington, DC, 1-124.

Roberts, J. A., \& Jones, E. (2001). Money Attitudes, Credit Card Use, and Compulsive Buying among American College Students. The Journal of Consumer Affairs, 35(2), 213-240.

Ruiz, D. C. (2003). Modigliani: Sesenta años de teoría económica. Economía Informa, 321, 21-29.

Schiffman, L. G., \& Kanuk, L. L. (2004). Consumer Behavior. Pearson Education International.

Villeda, S. M., y Nava, B. I. (2019). Hipótesis del ciclo de vida en México: un análisis del ingreso por género. Problemas del Desarrollo, 50(197), 3-29.

Yamauchi, K. T., \& Templer, D. J. (1982). The development of a money attitude scale. Journal of Personality Assessment, 46(5), 522-528.

Zaiunul, A. A. (2017). The Influence of Financial Knowledge, Control and Income on Individual Financial Behavior. European Research Studies Journal, 635-648. 